\title{
Model-Based Sensitivity Analysis of Flapping Wing
}

\section{Drive-Line Parameters}

\author{
Siemen Timmermans ${ }^{\mathrm{a}}$, and Dirk Vandepitte ${ }^{\mathrm{b}}$ \\ KU Leuven, Celestijnenlaan 300, Box 2420, Leuven 3001, Belgium.
}

\section{Introduction}

Natural evolution has created flying animals and insects with wings flapping in a horizontal stroke plane to accomplish a very agile flight $[1,2]$. When studying nature, it becomes clear that this type of flight contains characteristics, useful for unmanned air vehicles (UAVs) that fly indoors or in confined spaces [3]. The class of small drones studied in this work is called flapping wing nano air vehicles (FWNAVs) [4]. The goal of FWNAV research is to mimic the advantages seen in nature to robotic flight by reproducing the insect wing motion [5-7]. The most important features of the wing motion are a reciprocating back and forth stroke motion at a high frequency and a rotation of the wing itself to strike the incoming air at an optimal angle, called the wing pitch [8]. Prototype artifacts with different sizes are in various phases of research and development. The smallest FWNAVs copy insects and bumblebees like the Harvard Robobee $[9,10]$. Bigger concepts are based on hummingbirds like the Aerovironment Nano Hummingbird [11], the Purdue robotic hummingbird [12] and the Kulibrie FWNAV [13], developed at KU Leuven.

FWNAVs need a specially designed drive-line to produce the insect wing motion. The stroke motion is the only motion that is actively driven in most FWNAVs. Qualification of FWNAVs with respect to the principle of stroke generation, leads to three main configurations. A first system uses a crank shaft mechanism to transfer a rotational motor motion to the high frequency back and forth flapping motion [14-16]. The motor works constantly around its optimal point of operation. The load on the motor is nonlinear and extra hardware is necessary to control the FWNAV stability.

\footnotetext{
${ }^{a}$ Ph.D. Researcher, Department of Mechanical Engineering, siemen.timmermans@kuleuven.be

b Professor, Department of Mechanical Engineering, Senior Member AIAA
} 
This system was developed for a first FWNAV prototype at KU Leuven [13]. Another configuration uses a direct transmission from the motor to the wing motion and the motor is driven with an alternating current $[12,17,18]$. This system reduces the complexity of the drive-line and therefore system robustness improves. Control can be exerted by actively adapting the motor current [19]. The introduction of an elastic element into the drive-line, which is designed to operate at a near resonance frequency is shown to reduce the overall power requirements of the system $[12,20]$. This concept is currently used in the Kulibrie FWNAV which serves as reference prototype. A third configuration uses a piezoelectric actuator [21]. This concept is particularly effective for very small designs but a DC-DC converter is needed, which poses a significant weight challenge.

Improvement of overall performance is essential to increase the use of FWNAVs in practical applications. This can be done by optimizing the wing design and aerodynamics [22, 23] or by optimizing the drive-line dynamics. Because the wing stroke is the most demanding motion of the system, this work focuses on the wing drive-line dynamics, driving the stroke motion to accomplish this performance gain. The study uses a model-based approach to complement the foregoing experimental work. This model-based approach starts from a lumped parameter single degree of freedom (DOF) mass-spring-damper model, which is adopted by several research groups $[12,15,18,20,24-$ 26]. This model concept is used in the assumption that the influence of the wing pitch dynamics on the stroke dynamics is negligible. However the change in wing inclination is in previous research always included in the calculation of the wing aerodynamics. This work starts from the idea that $1 \mathrm{DOF}$ is sufficient to model the relevant phenomena present in the drive-line dynamics and it completely removes the model terms related to the second DOF [27]. The input to the resulting second order nonlinear time-invariant model is the motor current. The model simulation provides an instantaneous stroke angle.

A sensitivity analysis using the resulting model expression identifies the parameters which have the largest influence on performance. The efficiency of the drive-line system is used as the key performance indicator. This paper distinguishes between wing motion parameters and drive-line system parameters. Sensitivity analysis of the wing motion parameters gives the optimal controller input settings. Efficiency analysis of the drive-line system parameters is utilized to predict the 
optimum settings for the physical drive-line components.

An experimental set-up supports the model-based approach, helping with model parameter identification and for validation purposes. The set-up consists of the Kulibrie FWNAV drive-line, complemented with measurement equipment. The pitch motion is mechanically blocked to physically represent the same situation as used in the mathematical model expression (1 DOF). The experimental set-up is used to demonstrate the accuracy of the model-based sensitivity analysis and a subset of the model parameters are validated on the physical set-up. The drive-line components that are experimentally adjusted in this work are system inertia (using weights) and wing area

(using a set of scaled wings). In addition, the stroke motion amplitude and the flapping frequency can be set in the controller.

The paper starts with an explanation of the wing drive system. The mathematical model equation is introduced in the next section together with the assumptions on which the model is based. Afterwards, the experimental set-up with its modularity is introduced and the parameter identification procedure is explained. The results of the paper are subdivided in two sections: the model validation and sensitivity analysis. The first section discusses the numerical accuracy of the model simulations with a focus on possible sources of modeling errors. The paper concludes with model-based sensitivity analyses on the effect of parameter variations on the performance of the FWNAV.

\section{Materials and methods}

\section{A. Flapping wing drive-line}

\section{Drive-line system}

In order to generate the necessary forces to keep the FWNAV in the air, the wings make a very fast reciprocating motion with a flapping frequency on the order of 20 to $25 \mathrm{~Hz}$ and wing tip velocity on the order of $10 \mathrm{~m} / \mathrm{s}$. When looking at the wing motion of insects and small birds, two main motion components are identified: a horizontal motion with the wing gliding through the air, called the wing stroke motion, and a rotation of the wing around the main spar to obtain an optimum inclination angle, $\alpha=90-\eta$, relative to the local air stream, called the wing pitch motion (both 


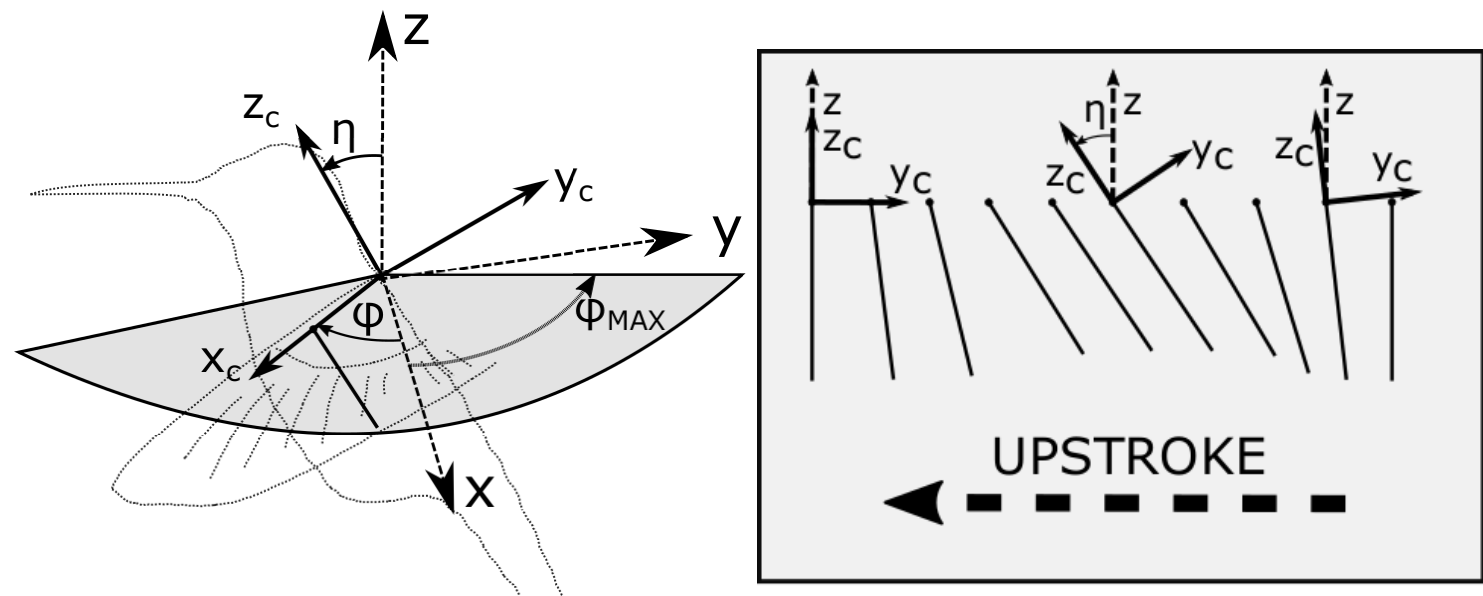

b)

a)

Fig. 1 Flapping wing motion in reference frame $x y z$ and co-rotating frame $x_{x} y_{c} z_{c}$.

a) Stroke motion indicated by shaded stroke plane with stroke angle $\phi$, and amplitude $\phi_{\max }$ b) View in $x_{c}$ direction, showing wing pitch motion, indicated by angle $\eta$.

components are shown in Fig. 1). When mimicking small birds and insects, the two main wing motions are copied mechanically.

The wing stroke motion (indicated in Fig. 1a) is the main component in FWNAVs. Ideally, the wing motion is sinusoidal $[18,26,28]$ and it can be characterized by stroke amplitude, $\phi_{\max }$, and frequency, $f$. The maximum amplitude is physically limited by system design. Frequency is a design parameter of the system, which can be operated at a range of frequency settings. The physical characteristics of the wings used in the reference prototype set this frequency range and experiments are done in the range of $16 \mathrm{~Hz}$ to $26 \mathrm{~Hz}$. A direct drive system actively drives the wing stroke motion, as shown in Fig. 2a. An elastic element supports the reciprocating motion by storing energy in a part of the cycle and releasing it afterwards. Figure $2 \mathrm{~b}$ shows a simplified scheme of a direct drive system with the system modelled as a discrete mass-spring-damper system. A DC motor drives the system with a sinusoidal input torque. A gear transmission increases motor torque. The main contribution to the total system inertia originates from an oscillating wing fixed at the end of the drive-line. The elastic element is mounted between the gear transmission and the load. The main contribution to the damping originates from the aerodynamic drag on the load and from 
friction in the system. System output is a flapping motion at the same frequency as the sinusoidal input and with a stroke amplitude depending on the amplitude of the input current.

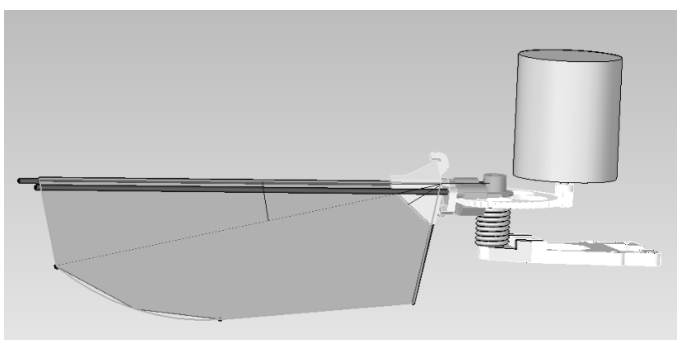

a)

Fig. 2 a) CAD drawing of the direct drive system. b) Simplified schematic for a generic direct drive system.

\section{BL: Friction \\ KS: Spring stiffness \\ N: Gear ratio \\ $\varphi$ : Stroke angle \\ I: Motor input current \\ JW: Wing inertia \\ BAERO: Wing damping}

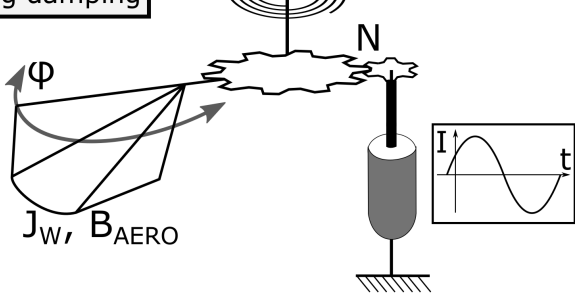

b)

The wing pitch motion (shown in Fig. 1b) is not actuated in the reference prototype, which is similar to most FWNAVs $[8,15,29]$. The wing is free to rotate as a result of the aerodynamic forces acting on the wing, inertial forces of the wing, and restoring forces from an elastic element. As for the pitch motion, no energy is added to the system and the pitch dynamics have very little influence on the stroke dynamics, this work studies a drive-line with a wing that is fixed as far as the pitch motion is concerned. This configuration removes one DOF and fixes the value $\alpha=90^{\circ}$

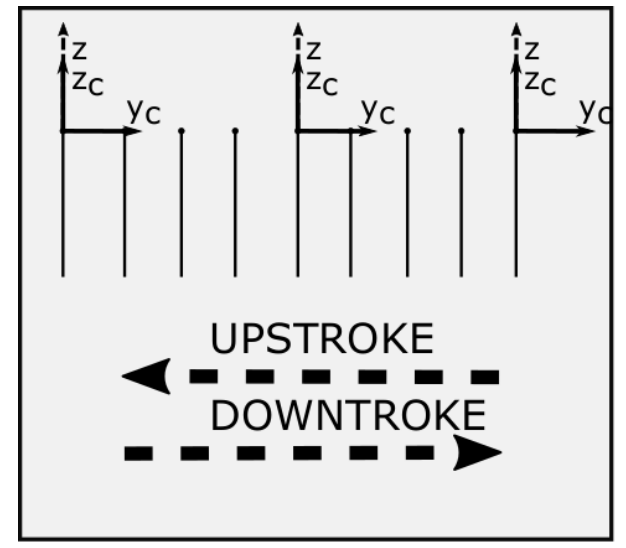

Fig. 3 Wing chord in $x_{c}$-direction showing fixed inclination angle. 
(Fig. 3 shows a wing chord in the $x_{c}$ direction, in up- and down-stroke). The wing only produces drag which is sufficient to represent the damping in the drive-line.

\section{Oscillating wing}

Wing design has an important effect on system performance. In addition to the obvious parameters of wing shape and wing dimensions previous research has shown that wing stiffness in transverse deflection and torsion has a significant effect too [30]. In order not to overload the sensitivity analysis in this paper, only one wing shape is used, which results from an earlier experimental optimization process [31]. The wing is designed to be as stiff as possible, and assumed to be rigid in the further analysis. This results in a constant $\eta$ over the wing span in the $x_{c}$ direction of the co-rotating frame. Thin carbon fiber composite rods make up the wing frame. They join at the base of the wing, called the wing root. A Mylar membrane is attached to the rods. Figure 4 shows the wing complemented with the geometric wing parameters: the length of the leading edge $R$, the chord length $c$ as a function of the running coordinate $\mathrm{r}$ along the leading edge, and the associated mean chord length $\bar{c}$.

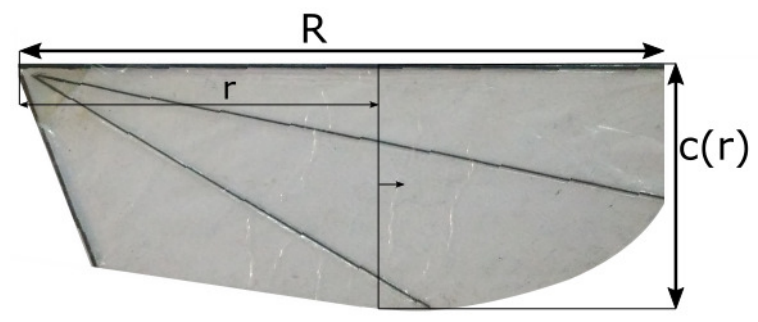

Fig. 4 Wing shape and location of wing veins.

\section{B. Dynamic modeling approach}

This section introduces the mathematical model that describes the wing drive-line dynamics. State of the art research uses the expression for a 1-DOF, adapted mass-spring-damper system with the mass modeled as a beam damped by wing aerodynamics [25]. There are three important assumptions in the modeling approach.

1. The 1 DOF model assumes that the stroke is the main motion and that the wing pitch dynamics 
have a negligible effect on the stroke dynamics.

2. The translational drag component calculated from quasi-steady theory [29] suffices to incorporate the wing damping. The equation does not include the unsteady phenomena that occur in flapping flight. The drag coefficient is identified using an experimental procedure (see section II C 3).

3. The stroke angle varies with time in a sinusoidal way. Experimental measurement of the stroke angle confirms that the motion is indeed sinusoidal. The full line in Fig. 5 shows raw stroke angle data, measured on the set-up (details in section II C 1), and the dotted line gives a sine fit.

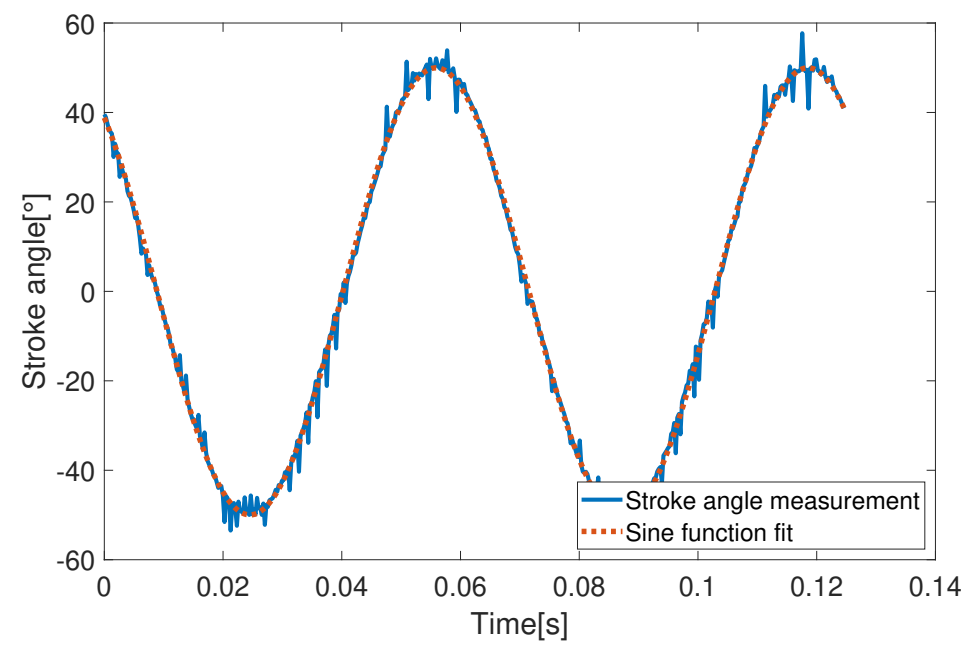

Fig. 5 Validation of the sinusoidal stroke angle assumption.

The mathematical formulation for the mass-spring-damper system driven by a DC motor is

$$
\begin{gathered}
J_{s} \ddot{\phi}+B_{l} \dot{\phi}+B_{A E R O}|\dot{\phi}| \dot{\phi}+K_{s} \phi=N K_{i} i \\
V_{m}=R i+K_{e} N \dot{\phi}
\end{gathered}
$$

Equation (1) is a second-order nonlinear time-invariant model expression as a function of the instantaneous stroke angle $\phi$ and the first and second time derivative of the stroke angle, $\dot{\phi}$ and $\ddot{\phi}$. 
The right-hand side gives the driving torque, proportional to the DC motor current $i$. The left-hand side shows the usual terms in mass-spring-damper systems, complemented with a term for the wing aerodynamics. $J_{s}$ is the total inertia of the system calculated as $J_{s}=J_{L}+J_{G}+N^{2} J_{m}$ with $J_{L}$ the load inertia, $J_{G}$ the gear inertia, $J_{m}$ the motor inertia and $N$ the gear ratio. $B_{l}$ is the total linearized damping, which is identified in the section on parameter identification (section II C 3). $B_{A E R O}$ is the aerodynamic damping calculated with the theory of quasi-steady aerodynamics as $B_{A E R O}=0.5 R^{4} \bar{c} r_{3}^{3} \bar{C}_{D}(\alpha)$ with $\bar{C}_{D}$, the mean drag coefficient, identified in the section on parameter identification. $K_{s}$ is the spring stiffness in [units of $\mathrm{Nm} / \mathrm{rad}$ ]. $K_{i}$ is the motor torque constant. When the inductance is negligible, the DC motor equation can be written as in Eq. (2) [12], and it is used to calculate the voltage $V_{m}$ over the terminals. $R$ is the motor resistance. $K_{e}$ is the back-EMF constant.

The mathematical expressions are solved using Mathworks Simulink. The input is a sinusoidal expression for the current with a prescribed amplitude and frequency. Model output is an instantaneous stroke angle oscillating at the same frequency and a voltage amplitude.

\section{Experiment drive-line system}

\section{Measurement set-up}

An experimental drive-line set-up complements the theoretical analysis and it is used to identify model parameters and to validate model results. The set-up uses a copy of the mechanical wing driveline system of the flapping wing reference prototype. Figure 6a presents the drive-line in the set-up together with drive electronics, sensors and measurement electronics. A schematic representation is given in Fig. 6b.

A Nordic NRF52 microcontroller is the brain of the set-up. It controls the signal to the motor via output pins, and reads in the data of the different sensor modules via an analog-to-digital converter (ADC) with a sample frequency of $15 \mathrm{kHz}$ per channel. 5 seconds of sensor data are stored internally on a chip. A PmodHB3 low current bridge module drives the motor using a a pulse-width modulated (PWM) signal to control the motor current. A Hall effect alternating current sensor, model L18P003S05, measures the instantaneous motor current. The range of the sensor is $\pm 3 \mathrm{~A}$ and 


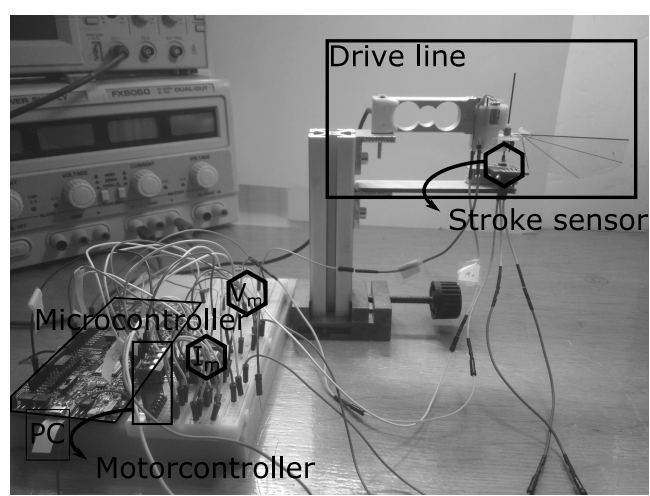

a)

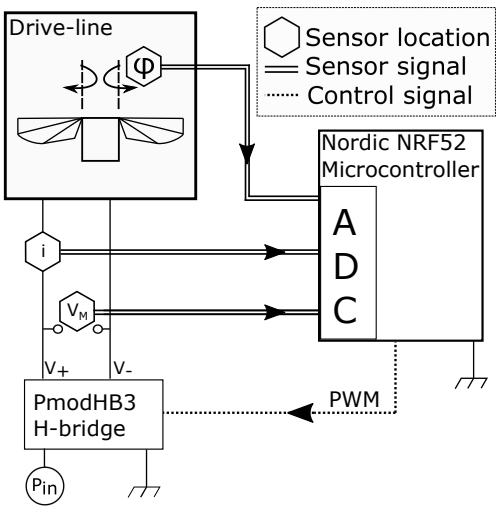

b)

Fig. 6 Set-up with location of sensors, drive electronics and microcontroller. a) Photo of the real set-up. b) Schematic showing the connections.

an analog output voltage is directly read by the ADC. Taking into account the ADC resolution of $14 \mathrm{bits}$, this results in a theoretical accuracy that is smaller than $1 \mu \mathrm{A}$. The current measurement is done in a series configuration with the motor, immediately in front of the motor terminals. The current measurement results are validated using another current sensor (model ACS712). The ADC inputs of the microcontroller measure the instantaneous voltage over the motor terminals. A Hallbased rotary magnetic position sensor, model AS5600, measures the instantaneous stroke angle output. The sensor outputs an analog voltage to the ADC which gives $0.02^{\circ}$ accuracy. A small circular diametric magnet is mounted on the stroke axis, moving with the wing, which allows to read the rotary position. Results are validated using a slow motion camera. Time is measured with the microcontroller $16 \mathrm{MHz}$ clock and synchronized with the different measurements. The flapping frequency is calculated from the measured time and validated using a stroboscope. The controller allows us to easily change the actuation frequency of the flapping motion and the input current. This setting determines the amplitude of the stroke motion and the frequency of the stroke motion.

A BCM 1668(S) single point load cell is used in the experiments that require a force measurement. This load cell measures the vertical force during flapping. Measured forces are averaged over a full flapping cycle. 


\section{Different drive-line configurations}

The drive-line is built in a modular manner to accommodate adaptation of the physical driveline parameters. The parameters that can be changed are listed in Table 1, column 1 . The modelbased sensitivity analysis in this work covers all system parameters, except the motor parameters (indicated in the second column). Only a subset of the parameters are also experimentally adapted in this work to validate results (listed in the last column).

Table 1 Drive-line parameters.

\begin{tabular}{lcc}
\hline \hline Parameter & $\begin{array}{c}\text { Changed in } \\
\text { model simulations }\end{array}$ & $\begin{array}{c}\text { Experimental } \\
\text { validation }\end{array}$ \\
\hline Wing size, $S$ & Yes & Yes \\
Total inertia, $J_{s}$ & Yes & Yes \\
Spring stiffness, $K_{s}$ & Yes & No \\
Gear ratio, $N$ & Yes & No \\
Friction, $B_{l}$ & Yes & No \\
Motor constants, $K_{i}, K_{e}, R$ & No & No \\
\hline \hline
\end{tabular}

The wing is discussed in section II A 2. Several wing sizes are used in the test campaign but the description in this paper is limited to a subset of two wings. The values for the leading edge length $\mathrm{R}$ and the wing planform area $S$ are listed in Table 2, together with other important wing characteristics. The third column gives the rotational moment of inertia $J_{L}$ of the wing around the wing root. The last column of Table 2 shows the natural frequency $f_{0}$ of the wings in the reference

Table 2 Wing properties.

\begin{tabular}{ccccc}
\hline \hline Wing ID & \multicolumn{5}{c}{ S[mm $\left.{ }^{2}\right]$} & LE[mm] & $\mathrm{J}_{W}\left[\mathrm{kgm}^{2}\right]$ & $\mathrm{f}_{0}[\mathrm{~Hz}]$ \\
\hline Wing 1 & 1293.27 & 63.30 & $3.64 \mathrm{e}-07$ & 19.81 \\
Wing 2 & 1161.94 & 60.00 & $3.40 \mathrm{e}-07$ & 20.21 \\
\hline \hline
\end{tabular}


set-up, calculated with Eq. (3) for a simple 1 DOF mass-spring system.

$$
. f_{0}=\sqrt{\frac{K_{s}}{J_{s}}}
$$

The total system inertia $J_{s}$ affects the resonant frequency of the system, as given by Eq. (3). A variation in inertia can be caused by a number of factors, e.g. wing design, gear material, etc. The effect is simulated by adding a small mass outside of the center of rotation of the system which increases the rotational inertia. The effect of spring stiffness $K_{s}$ is similar to the effect of $J_{s}$. Springs with different stiffness constants can be used to accomplish this, but a number of different springs need to be ordered and installed. Here, the real advantage of a model-based approach comes forward and this parameter is only studied using simulations. The same is true for an adaptaion of gear ratio $N$ or damping $B_{l}$.

\section{Parameter identification}

This section defines numerical values for the model parameters in the simulations. The quality of the model simulations depends largely on the accuracy of the model parameters. The model uses two types of parameters: parameters that are a priori known and parameters that are found from an inverse parameter identification procedure. The first type is calculated from the respective datasheets of the drive-line components and simple analytical expressions. Parameters of the second type need an identification procedure in order to extract their numerical values. The numerical values of these parameters remain constant during a single series of experiments but depend on the external conditions of the set-up and vary whenever small physical variations occur in the set-up. This makes an inverse parameter identification procedure the quickest and most accurate way to quantification.

The motor is a $7 \mathrm{~mm}$ brushless DC motor with $2.6 \mathrm{~g}$ mass with a terminal resistance of $1.4 \Omega$, a torque constant of $0.81 \mathrm{Nmm} / \mathrm{A}$, and a rotor inertia of $5 \cdot 10^{-9} \mathrm{kgm}^{2}$. The gear transmission is custom made for the set-up with a gear ratio of 10 . The springs are tested over the entire range of operation for linearity and accuracy of the spring constant $K_{s}$. Its value $0.0098 \mathrm{Nm} / \mathrm{rad}$ is taken from the datasheet with no need for further updating. The aerodynamic damping is calculated 
using a quasi-steady aerodynamic model. The drag coefficient in the equation is an overall system parameter which remains unknown. The next paragraph explains the experimental procedure to identify this parameter as well as the total linear damping and the total inertia of the system.

One frequency sweep experiment with constant current amplitude input suffices for this parameter identification procedure. Constant current amplitude implies that a constant torque amplitude is applied to the drive-line. The controller actuates the drive-line for 5 seconds at every frequency in the frequency range, with a frequency step of $1 \mathrm{~Hz}$. Actuation for a duration of five seconds is proven to be sufficient to eliminate the effects of possible transients. The natural frequency is extracted from the experimental data using the phase difference between current input and stroke angle output and the total inertia is calculated, using Eq. (3). After application of the same constant input to the model in Eq. (1), the remaining parameters can be identified. The simulation uses an estimated value as a starting point for these parameters. The calculated stroke angle values are compared with the experimentally measured values. The damping coefficient is updated to make the simulations match the experimental data over the entire frequency range.

\section{Model validation}

This section verifies the accuracy of the model simulation results by means of a frequency sweep experiment. Application of the same input current to the mathematical model and the experimental set-up is used to compare the experimental stroke angle output with the simulated stroke angle output. Two frequency sweep experiments are performed. The configuration uses two stiff wings connected to allow 1 DOF motion. The model is tuned to represent the two wing configurations.

Figure 7 shows the comparison between the experimental frequency sweep and the resulting model simulations. The curves have a similar pattern. The addition of the elastic element which supports the motion results in a peak amplitude which is flattened due to the aerodynamic damping of the wing in the system. The peak amplitude occurs at a frequency which is slightly below the theoretical resonance frequency (given in Table 2). The deviation is larger for a larger wing, wing 1 , and the model error increases when the frequency of operation increases above the peak amplitude frequency. This observation indicates that the assumption of quasi-steady aerodynamics is an important source of modeling error. Also, the influence of the increasing flexibility with 


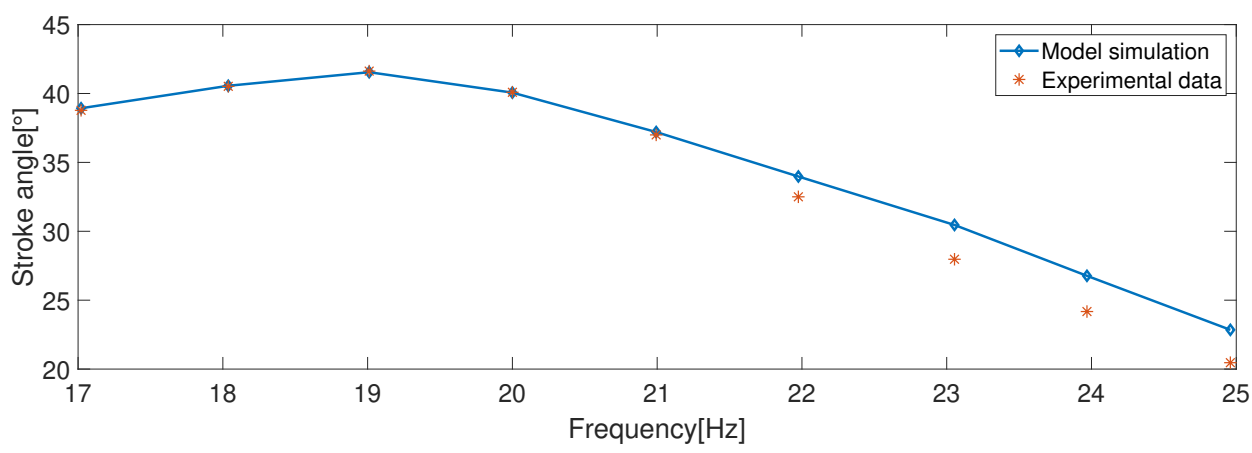

a)

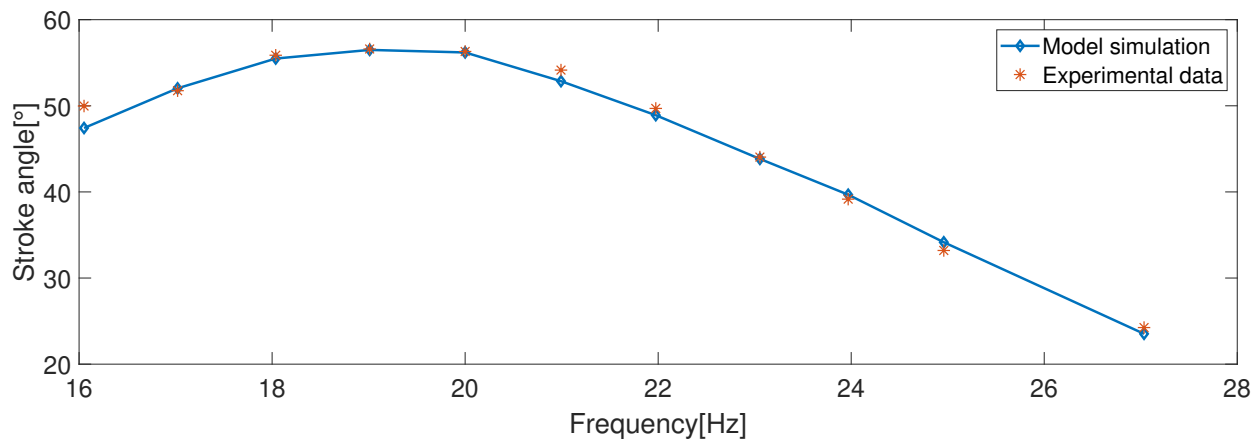

b)

Fig. 7 Constant input frequency sweep, comparison between simulations and experimental data for the adapted model. a) Wing 1 moving with 1 DOF. b) Wing 2 moving with 1 DOF.

increasing wing size is a factor which contributes to the deviation between model and experiment.

The agreement between model and experiments is observed to be fair and it is the motivation to use this model for sensitivity analysis. The absence of lift force is the main challenge when using the described set-up because lift force generation is the ultimate goal of a flapping wing drive-line system. Limited influence on the results of the sensitivity analysis is expected as aerodynamic drag is present and this introduces the same second order damping term in the model which would also be applicable for a regular 2 DOF flapping wing system. To ensure that results can be generalized to a regular drive-line, this work proposes a solution using a generalized velocity parameter, defined as the mean velocity of the center of pressure of the wing and incorporating the wing size. The genenralized velocity parameter, $U_{C P}^{\prime}$, is given by 


$$
U_{C P}^{\prime}=S \hat{r}_{2} R 2 \pi f A
$$

where $S$ is the wing planform, $\hat{r}_{2} R$ is the location of the center of pressure and $2 \pi f A$ is the amplitude of the angular velocity. When using the regular 2 DOF flapping wing system, experiments show that a one to one relationship exists between the lift force and this generalized velocity parameter, on the condition that the wing shape, stiffness and maximal angle of attack don't change. This translates to the mathematical condition of a constant lift to drag coefficient ratio. Figure 8 gives the experimental lift force as a function of the generalized velocity parameter. The lift force measurement set-up is described in section II C 1 . Figure 8 shows wings with 4 different sizes, each tested at several (randomly chosen) stroke amplitudes and flapped at different frequencies in the range of $16 \mathrm{~Hz}$ to $26 \mathrm{~Hz}$. Calculation of the generalized velocity parameter and comparison of this value to the lift force experimentally proves the relationship. Keeping a constant generalized velocity parameter when doing an analysis allows use of the proposed model and bypasses the challenge that the absence of lift force introduces.

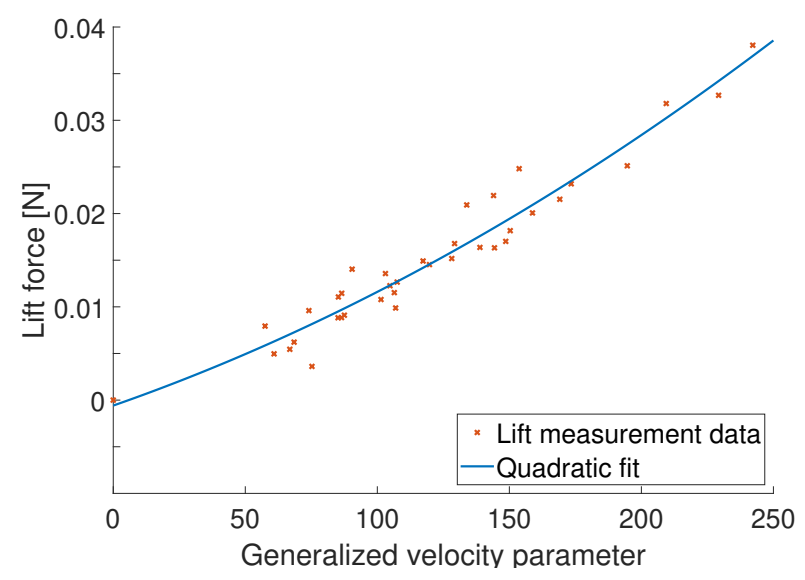

Fig. 8 Experimentally measured lift force for different values of the generalized velocity parameter.

\section{Sensitivity analysis}

Parameter sensitivity analysis identifies the parameters with the largest influence on the performance in support of better design decisions. The drive-line model is a powerful tool for sensitivity 
analysis. After the model is validated, it simulates the drive-line behavior without the need for additional extensive experimental testing. The following material uses the 1 DOF drive-line as described in section II A and the associated model described in section II B.

An appropriate performance parameter needs to be selected for the dynamic analysis of the drive-line. This work uses an indicator of efficiency for comparison of the different drive-line with wing configurations. Efficiency of FWNAVs is defined as the ratio of the useful power from the generated force of the wings over the input power at the motor terminals [18]. The useful power generated by the wings is calculated as the total aerodynamic torque multiplied with the wing angular velocity. The input power is calculated using the motor equation (2).

$$
\eta=\frac{P_{\text {out }}}{P_{\text {in }}}=\frac{B_{A E R O} \dot{\phi}}{V_{m} i}
$$

The dynamic analysis distinguishes two types of parameters in the drive-line system: wing motion parameters and system parameters. The wing motion parameters for a hovering flapping wing are the frequency and the amplitude. Section IV A studies their effect on the efficiency of the flapping wing. Independent setting of wing motion parameters is possible for every experiment using the controller which adapts the wing motion parameters for every simulation. The drive-line system parameters are the total inertia, the gear transmission ratio, the spring stiffness, the wing size and the frictional damping. The parameter sensitivity analysis of section IV B sets these system parameters individually while holding the other system parameters constant. The model is used for an analysis over a range of parameter values, including upper and lower limit values. Efficiency analysis of every wing motion parameter, of the wing inertia and of the wing size is experimentally validated with the objective of supporting the conclusions drawn with the model and for further validation of the model-based approach. This paper uses constant motor parameters. Motor selection is done in other works $[24,25]$. 


\section{A. Wing motion parameters}

\section{Frequency}

The choice of the right operational flapping frequency minimizes energy consumption of the system. Previous research shows two frequencies which deserve special interest when doing a frequency analysis of an oscillating drive-line: the peak frequency and the natural frequency [26]. The peak frequency maximizes the amplitude of the stroke angle for a constant motor input torque. The natural frequency is calculated as the ratio of the total inertia over the spring stiffness, using Eq. (3), and it is a characteristic of system design (calculated in Table 2). The peak frequency is lower than the natural frequency in strongly damped systems. The range for the frequency sensitivity analysis contains both frequencies. The generalized velocity parameter and hence the amplitude of the wing velocity is held constant for the entire frequency range. The analysis shows that flapping the wings at the system natural frequency maximizes the efficiency (Fig. 9) — which does not come as a surprise — but this observation is also shown to be independent of the wing velocity. The analysis uses model formulation (1) and validation using the experimental set-up confirms the result. The result is consistent with the results of Zhang et al. [26].

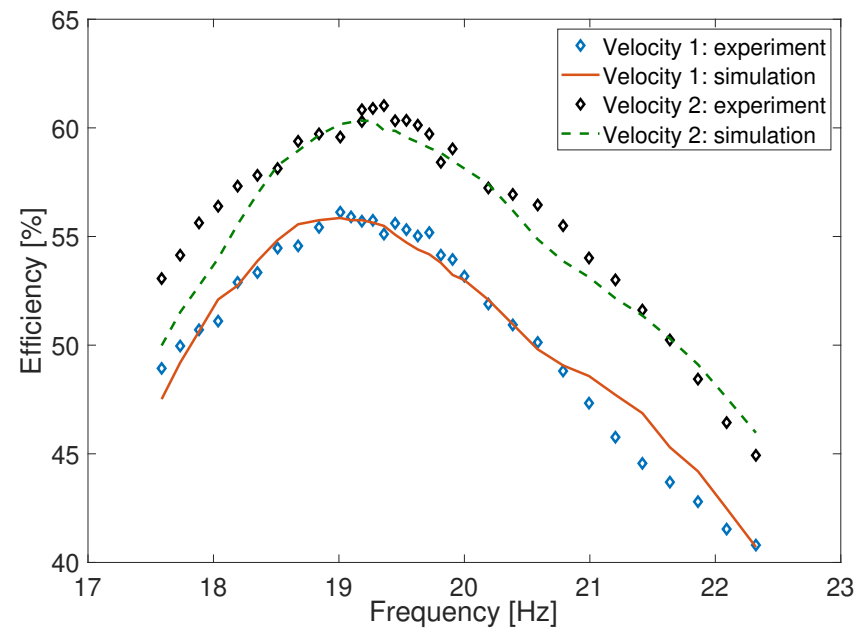

Fig. 9 Variation of the efficiency as a function of the operational flapping frequency. 


\section{Stroke angle amplitude}

The stroke angle for a fixed flapping frequency affects wing velocity. Wing velocity has a direct link to lift production of the flapping wings and for this reason the sensitivity analysis of the stroke angle amplitude is converted to a sensitivity analysis of the wing velocity. Figure 10 shows the effect of a change in wing velocity on the efficiency using the model approach. Experimental data serve as validation of the conclusions and the model-based approach.

The figure shows that maximization of the velocity amplitude results in highest efficiency for a chosen wing. An optimum exists far beyond the maximum velocity that is physically feasible in the current drive-line configuration after which efficiency decreases again. The motor characteristics given in the motor datasheet explain the existence of this optimum wing velocity and optimal motor selection (see ref. [24]) does have an influence on the efficiency.

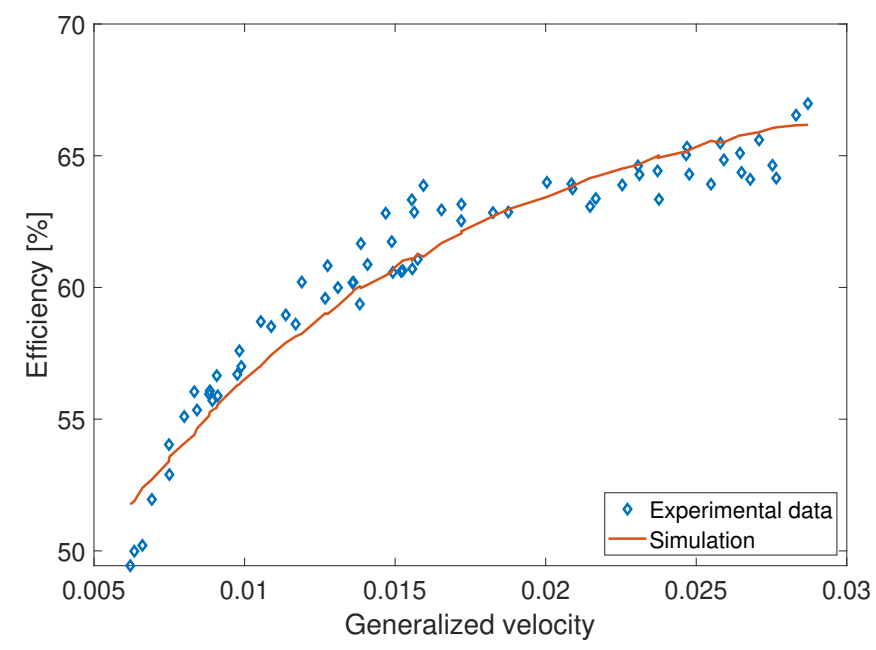

Fig. 10 Variation of the efficiency as a function of the generalized velocity parameter.

\section{B. Drive-line system parameters}

The analysis of this section varies one drive-line system parameter while keeping the other system parameters constant. The two wing motion parameters on the other hand are not constant between the experiments and they are used to have the system operate at its optimal frequency and stroke angle amplitude. Manual setting of the controller is possible and choice of the numerical 
values for these parameters is done in accordance with the conclusions of the previous section.

The first conclusion is that efficiency is optimized by tuning the drive frequency to the system natural frequency. If the system drive-line parameter under investigation has an influence on the natural frequency of the system by adapting the stiffness of the elastic element or the total rotational inertia, the adapted natural frequency must be calculated and every single simulation must be performed on the adapted resonance frequency. The effect of flapping off-resonance is detrimental to the efficiency and can lead to misinterpretation of the results.

The second conclusion is that a constant value for the generalized wing velocity parameter is necessary to compare the different experiments in an objective way. This is done in practice by tuning the value for the stroke angle amplitude in the experiments (for a specific frequency, the relationship between velocity amplitude and stroke angle amplitude is linear).

\section{Rotational inertia}

The rotational inertia parameter changes the natural frequency of the system as specified in Eq. (3) and thus the operational flapping frequency. A higher rotational inertia leads to a lower resonance frequency and vice versa. A change in frequency with a constant stroke amplitude changes the wing velocity, which is unwanted. This analysis eliminates the influence on the wing velocity

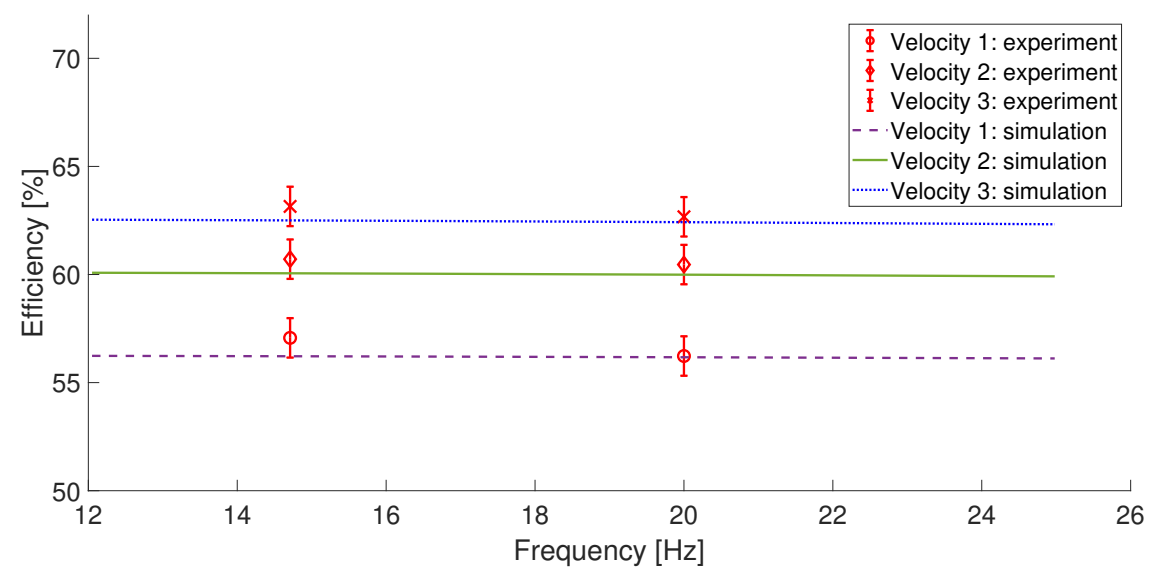

Fig. 11 Sensitivity of the drive-line efficiency to the inertia. The horizontal axis shows the adapted resonance frequency resulting from a change in inertia. 
by adapting the wing stroke amplitude when the frequency changes. Figure 11 shows the change of the efficiency as a function of the rotational inertia for three different values for the wing velocity. The graph demonstrates that a change of the system inertia does not change the efficiency of the drive-line operation, which leads to horizontal lines in the graph. Minor dependence is observed of the efficiency to the frequency but this is smaller than experimental accuracy and can be neglected. Experiments confirm the analysis. Only experiments with increased rotational inertia are performed because of practical considerations (the basic configuration in the set-up has minimal inertia so it is only possible to add mass/inertia). Figure 11 shows the experimental result in combination with error bars. The error bar is calculated using 125 experiments and associated simulations. The absolute difference between the simulated result and the experimentally measured result is calculated and the mean value is taken as error bar. It gives the expected deviation of the experimental data with respect to the model simulations.

\section{Elastic element stiffness}

The analysis of the elastic element stiffness is similar to the one of the rotational inertia, be it with inverse infects, based on Eq. (3). The parameter affects frequency and velocity and these are changed accordingly in the simulations. The change of the efficiency as a function of the frequency of the system, calculated from the elastic element stiffness, evolves very similarly to what is shown in Fig. 11. The stiffness value does not change the efficiency of the drive-line operation in a way that would make a noticeable difference in the frequency range of interest and the graph is therefore omitted.

\section{Gear transmission ratio}

The gear transmission plays a vital role when looking at torque and power transfer in the driveline system. In addition to the fact that the motor needs to be able to deliver the necessary torque and power, it also makes sense to look at the influence of the gear ratio on the efficiency. Figure 12 shows the influence of the gear transmission ratio on the efficiency using the model approach. 


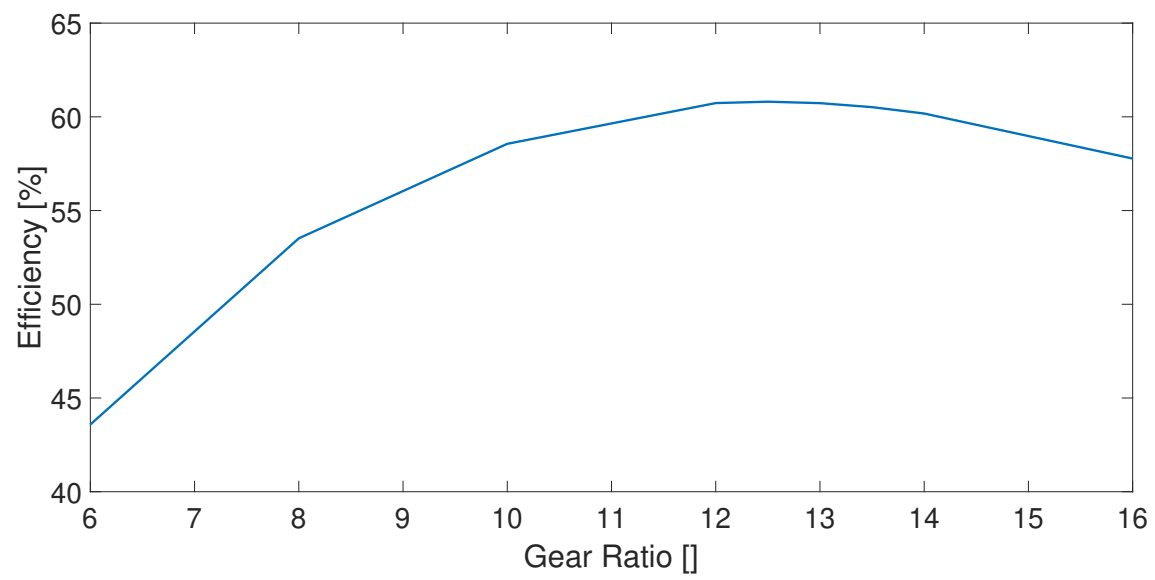

Fig. 12 Sensitivity of the drive-line efficiency to the gear ratio.

Experimental validation is again not feasible for the similar reasons of practical implementation as explained in the previous section. The graph shows a clear optimum value for the gear ratio that can be explained physically by looking at the motor datasheet. The datasheet shows that motor efficiency strongly depends on the motor velocity and driving torque and the transmission ratio affects these parameters. This observation explains the opportunity to shift the working point of the motor closer to its optimal working point by adapting the value of the transmission ratio.

\section{Wing size}

The analysis of the sensitivity of the efficiency with respect to the wing size only studies similar wings with different sizes. The ratio of lift coefficient over drag coefficient remains unchanged in this case which makes it possible to work with the constant generalized velocity parameter. This is a necessary condition for the analysis as the different sized wings then generate equal lift. In addition to the two wings described in Table 2, two extra wings are included in the analysis with the same wing shape and a larger wing size. Figure 13 shows the efficiency as a function of the wing size. A reduction of the wing size increases the efficiency, independent of the generalized velocity parameter. Experimental data confirm this conclusion. Two experimental data points are used for every wing (one for each value of the generalized velocity parameter), together with an error bar based on the scatter over the previous experimental results (calculated in the same way as the error 


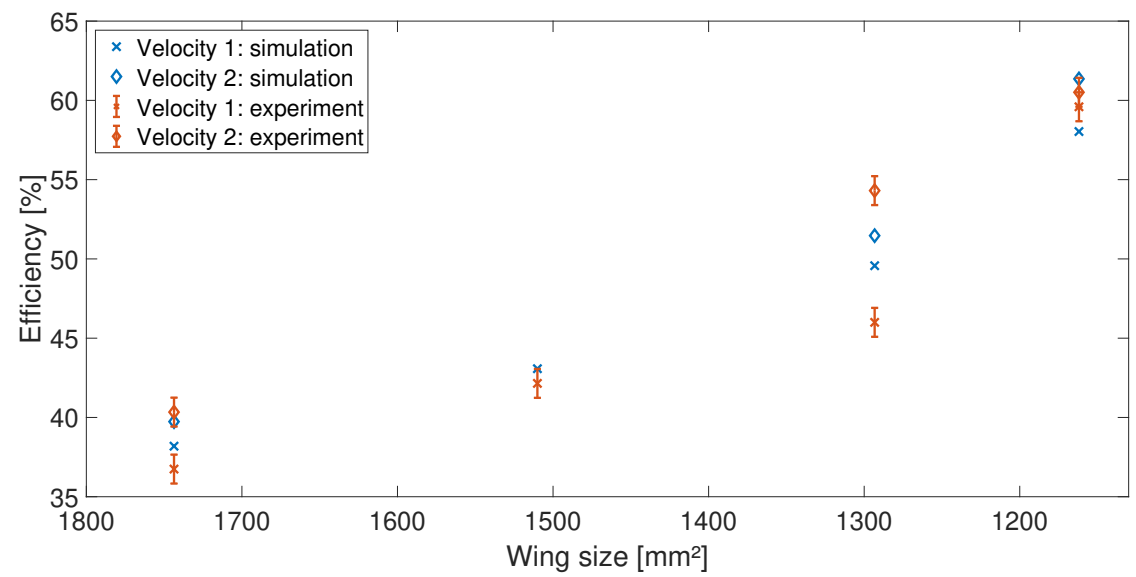

\section{Fig. 13 Influence of the wing size on the drive-line efficiency for two values of the generalized velocity parameter.}

bars in Fig. 11). Simulation results are not entirely within the specified experimental interval in every data point but the same trend is observed. The simplified aerodynamic expression used in the model is one of the reasons for the accuracy issues. Small imperfections in the fabrication of the wings are a second reason for the inaccuracies.

This analysis shows a positive effect of miniaturization of the wing on the efficiency. Together with the general tendency towards smaller designs for UAVs, this is an interesting intermediate conclusion. It demonstrates the potential of flapping wings in these smallest designs.

\section{Damping}

The sensitivity analysis of the frictional damping in the bearings may seem trivial as less damping is usually preferred. Figure 14 confirms this relationship between damping and efficiency. Minimization of damping traditionally comes with a cost, f.e. changing gear material can reduce damping but the cost is a mass increase. The model is used to calculate the acceptable cost for a specific reduction in damping. Of-the-shelf solutions for certain components can be compared with these results which leads to better component selection. 


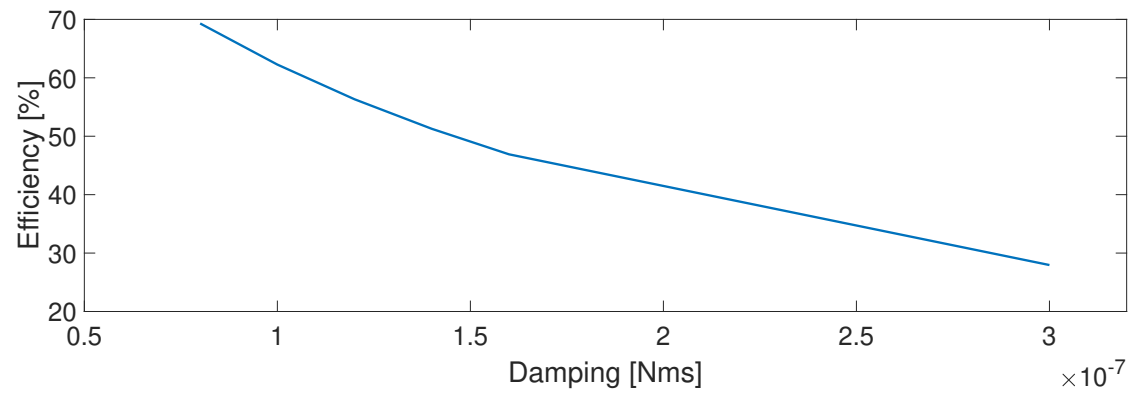

Fig. 14 Sensitivity of the drive-line efficiency to the frictional damping.

\section{Conclusion}

This paper demonstrates the use of a mathematical model expression for a parameter sensitivity analysis of the drive-line of a FWNAV. The expression for a second-order nonlinear time-invariant system describes the physical drive-line. This work uses a model describing a flapping wing drive-line set-up that is deliberately constrained to 1 DOF. The simulated stroke angle amplitude, calculated using the model expression, matches well with experimental data.

A sensitivity analysis of the drive-line targets system efficiency as a prime performance attribute. The objective of the analysis is to quantify the influence of the drive-line parameters on the system performance aiming at improved design decisions. The analysis distinguishes between wing motion parameters and wing drive-line parameters. The wing motion parameters are set by the system controller and the sensitivity analysis of these parameters gives the optimal operational point. The analysis confirms that the optimum flapping frequency is the natural frequency of the system and that an increase of the wing stroke amplitude leads to an efficiency increase. The wing drive-line parameters are the physical system parameters and optimum value for the wing motion parameters is set in the controller, based on the results of the sensitivity analysis of these parameters. This eliminates the influence of other settings on the analysis. The paper shows that the effect of rotational inertia and the stiffness of the elastic element is negligible in the current system. Optimum gear ratio for the current drive-line with motor is defined. Minimization of wing size is beneficial for the drive-line efficiency which shows flapping wings have potential when miniaturizing the FWNAV. Also a reduction of damping improves the drive-line efficiency. 
Experimental validation of a substantial subset of the simulations shows that the same conclusions are valid for the model-based approach and from experimental testing. The model-based approach is quicker and can also be applied when physical adaptation of the set-up is not easily possible as was the case for the variation of the spring stiffness or the gear ratio. This makes the method valuable both in the design phase of FWNAVs and in a later phase for optimization purposes.

\section{Acknowledgements}

The authors gratefully acknowledge the financial support of FWO-Vlaanderen (project G0B55.13).

\section{References}

[1] Wang, Z. J., "Dissecting Insect Flight," Annual Review of Fluid Mechanics, Vol. 37, No. 1, 2005, pp. 183-210, doi: 10.1146/annurev.fluid.36.050802.121940.

[2] Hedrick, T. L., Tobalske, B. W., Ros, I. G., Warrick, D. R., and Biewener, a. a., "Morphological and kinematic basis of the hummingbird flight stroke: scaling of flight muscle transmission ratio," Proceedings of the Royal Society B: Biological Sciences, Vol. 279, No. 1735, 2012, pp. 1986-1992, doi: 10.1098/rspb.2011.2238.

[3] Wilkins, P., "Insect-like Flapping, the propulsion of micro air vehicles," Annex of PhD Dissertation: Some Unsteady Aerodynamics Relevant to Insect-inspired Flapping-wing Micro Air Vehicles, 2008, pp. 391-403.

[4] Petricca, L., Ohlckers, P., and Grinde, C., "Micro- and nano-air vehicles: State of the art," International Journal of Aerospace Engineering, Vol. April 2011, 2011, pp. 1-17, doi: 10.1155/2011/214549.

[5] Weis-fogh, T., "Energetics of hovering flight in hummingbirds and in drosophila," Journal of Experimental Biology, Vol. 56, No. 1, 1972, pp. 79-104.

[6] Dickinson, M. H., Lehmann, F. O., and Sane, S. P., "Wing rotation and the aerodynamic basis of insect flight." Science, Vol. 284, No. 5422, 1999, pp. 1954-1960, doi: 10.1126/science.284.5422.1954.

[7] Madangopal, R., Khan, Z. A., and Agrawal, S. K., "Biologically Inspired Design Of Small Flapping Wing Air Vehicles Using Four-Bar Mechanisms And Quasi-steady Aerodynamics," Journal of Mechanical Design, Vol. 127, No. 4, 2005, pp. 809-816, doi: 10.1115/1.1899690.

[8] Bergou, A. J., Xu, S., and Wang, Z. J., "Passive wing pitch reversal in insect flight," Journal of Fluid Mechanics, Vol. 591, October 2007, pp. 321-337, doi: 10.1017/S0022112007008440. 
[9] Teoh, Z. E., Fuller, S. B., Chirarattananon, P., Prez-Arancibia, N. O., Greenberg, J. D., and Wood, R. J., "A hovering flapping-wing microrobot with altitude control and passive upright stability," IEEE International Conference on Intelligent Robots and Systems, 2012, pp. 3209-3216, doi: 10.1109/IROS.2012.6386151.

[10] Wood, R., "The First Takeoff of a Biologically Inspired At-Scale Robotic Insect," IEEE Transactions on Robotics, Vol. 24, No. 2, 2008, pp. 341-347, doi: 10.1109/TRO.2008.916997.

[11] Keenon, M., Klingebiel, K., Won, H., and Andriukov, A., "Development of the nano hummingbird: A tailless flapping wing micro air vehicle," Proc. 50th AIAA Aerosp. Sci. Meeting Including New Horizons Forum Aerosp. Expo., Aerosp. Sci. Meetings, 2012, doi: 10.2514/6.2012-588.

[12] Zhang, J., Cheng, B., Roll, J. A., Deng, X., Yao, B., and Member, S., "Direct Drive of Flapping Wings under Resonance with Instantaneous Wing Trajectory Control," IEEE International Conference on Robotics and Automation (ICRA), 2013, pp. 4029-4034, doi: 10.1109/ICRA.2013.6631145.

[13] Leys, F., Reynaerts, D., and Vandepitte, D., "Outperforming hummingbirds' load-lifting capability with a lightweight hummingbird-like flapping-wing mechanism." Biology open, Vol. 213, No. 5, 2016, pp. 725-734, doi: 10.1242/bio.014357.

[14] Oppenheimer, M. W., Sigthorsson, D. O., Weintraub, I. E., Smith, T. J., Dawson, J. C., and Doman, D. B., "Development of a flapping wing mechanism that can produce lift greater than weight," $A I A A$ Guidance, Navigation, and Control (GNC) Conference, August 2013, pp. 1-15, doi:10.2514/6.20135106.

[15] Arabagi, V., Hines, L., and Sitti, M., "A simulation and design tool for a passive rotation flapping wing mechanism," IEEE/ASME Transactions on Mechatronics, Vol. 18, No. 2, 2013, pp. 787-798, doi: 10.1109/TMECH.2012.2185707.

[16] Baek, S. S., Ma, K. Y., and Fearing, R. S., "Efficient resonant drive of flapping-wing robots," 2009 IEEE/RSJ International Conference on Intelligent Robots and Systems, IROS 2009, 2009, pp. 28542860, doi: 10.1109/IROS.2009.5354725.

[17] Campolo, D., Azhar, M., Lau, G. K., and Sitti, M., "Can DC motors directly drive flapping wings at high frequency and large wing strokes?" IEEE/ASME Transactions on Mechatronics, Vol. 19, No. 1, 2014, pp. 109-120, doi: 10.1109/TMECH.2012.2222432.

[18] Azhar, M., Campolo, D., Lau, G.-k., Hines, L., and Sitti, M., "Flapping Wings via Direct-Driving by DC Motors," 2013 IEEE International Conference on Robotics and Automation (ICRA), 2013, pp. 1397-1402, doi: 10.1109/ICRA.2013.6630753. 
[19] Timmermans, S., Leys, F., and Vandepitte, D., "Model-based Evaluation of Control Roll, Pitch, Yaw moments for a Robotic Hummingbird," Journal of Guidance, Control, and Dynamics, Vol. 40, No. 11, 2017, pp. 2933-2939, doi: 10.2514/1.G002819.

[20] Hines, L., Campolo, D., and Sitti, M., "Liftoff of a motor-driven, flapping-wing microaerial vehicle capable of resonance," IEEE Transactions on Robotics, Vol. 30, No. 1, 2014, pp. 220-232, doi: $10.1109 /$ TRO.2013.2280057.

[21] Jafferis, N. T., Graule, M. A., and Wood, R. J., "Non-linear resonance modeling and system design improvements for underactuated flapping-wing vehicles," IEEE International Conference on Robotics and Automation (ICRA), 2016, pp. 3234-3241, doi: 10.1109/ICRA.2016.7487493.

[22] Oppenheimer, M. W., Sigthorsson, D. O., Weintraub, I. E., and Doman, D. B., "Wing design and testing for a tailless flapping wing micro air vehicle," AIAA Guidance, Navigation, and Control Conference, January 2017, pp. 1-26, doi: 10.2514/6.2017-1271.

[23] Nan, Y., Karásek, M., Lalami, M. E., and Preumont, A., "Experimental optimization of wing shape for a hummingbird-like flapping wing micro air vehicle," Bioinspiration and Biomimetics, Vol. 12, No. 2, 2017, pp. 1-16, doi: 10.1088/1748-3190/aa5c9e.

[24] Campolo, D., "Motor selection via impedance-matching for driving nonlinearly damped, resonant loads," Mechatronics, Vol. 20, No. 5, 2010, pp. 566 - 573, doi: 10.1016/j.mechatronics.2010.05.008.

[25] Zhang, J., Cheng, B., and Deng, X., "Instantaneous wing kinematics tracking and force control of a high-frequency flapping wing insect MAV," Journal of Micro-Bio Robotics, Vol. 11, No. 1-4, 2016, pp. 67-84, doi: 10.1007/s12213-015-0085-4.

[26] Zhang, J. and Deng, X., "Resonance Principle for the Design of Flapping Wing Micro Air Vehicles," IEEE Transactions on Robotics, Vol. 33, No. 1, 2017, pp. 183-197, doi: 10.1109/TRO.2016.2626457.

[27] Timmermans, S. and Vandepitte, D., "Model-based analysis of the dynamic behaviour of the wing drive line of a robotic hummingbird," Proceedings of ISMA2018 International Conference on Noise and Vibration Engineering, 2018, pp. 3427-3442.

[28] Weintraub, I. E., Sigthorsson, D. O., Oppenheimer, M. W., and Doman, D. B., "Kinematic selection for a tailless flapping wing micro-air vehicle," AIAA Guidance, Navigation, and Control Conference, January 2016, pp. 1-20, doi: 10.2514/6.2016-0892.

[29] Whitney, J. P. and Wood, R. J., "Aeromechanics of passive rotation in flapping flight," Journal of Fluid Mechanics, Vol. 660, October 2010, pp. 197-220, doi: 10.1017/S002211201000265X.

[30] Sigthorsson, D. O., Oppenheimer, M. W., Doman, D. B., and Weintraub, I. E., "Wing flexibility induced control reversal for flapping wing vehicles: Theoretical analysis," AIAA Guidance, Navigation, 
and Control Conference, January 2017, pp. 1-15, doi: 10.2514/6.2017-1272.

[31] Leys, F., The Eurotrochilus Mechanicus, a robotic hummingbird driven by a resonant flapping mechanism, Ph.D. thesis, University of Leuven, 2017. 